\title{
Fatores que influenciam a comunidade de peixes em dois ambientes no baixo Rio das Mortes, Planície do Bananal, Mato Grosso, Brasil
}

\author{
Eliete F. da Silva ${ }^{1}$; Cesar E. de Melo ${ }^{1} \&$ Paulo C. Vênere ${ }^{2}$ \\ ${ }^{1}$ Laboratório de Ictiologia e Limnologia, Departamento de Ciências Biológicas, Universidade do Estado de Mato Grosso. \\ Rodovia BR 158, km 148, 78690-000 Nova Xavantina, Mato Grosso, Brasil. E-mail: eti_silva@yahoo.com.br; \\ meloce@yahoo.com \\ 2 Departamento de Ciências Biológicas e da Saúde, Instituto Universitário do Araguaia, Universidade Federal de Mato \\ Grosso. Rodovia MT 100, km 3,5, 78698-000 Pontal do Araguaia, Mato Grosso, Brasil.E-mail: pvenere@uol.com.br
}

\begin{abstract}
Factors influencing the fish community in two environments in the lower Rio das Mortes, Bananal floodplain, Mato Grosso, Brazil. The characterization of the limnological variables and of the fish fauna of a open lake and of the main channel in the Rio das Mortes floodplain was conducted in order to compare these environments and indicate the influencing environmental factors of the fish communities in each one of them. A significative difference was observed between the open lake and the river channel in relation to the water transparency, higher in the open lake, and water velocity, higher in the river channel. Species diversity was also different between the sites, with significantly higher value in the open lake. In both envitonments the highest richness and diversity values were obtained during the low water period, and the relative density (CPUE) values in the rising water period. The environments were more similar during the wet season, in relation to both fish abundance and limnological characteristics. This happens due to the homogeneization of the water and habitat structure caused by flooding. The results indicate that the flood pulse, associated with water velocity, was the major driving force for the fish fauna structure in the environments sampled in this study.

KEY WORDS. Diversity; flood pulse; limnology; similarity.
\end{abstract}

RESUMO. A caracterização limnológica e da ictiofauna em uma enseada e no canal principal do Rio das Mortes na sua região de planície de inundação foi feita com o intuito de comparar estes ambientes e indicar os possíveis fatores ambientais que influenciam as comunidades de peixes em cada um deles. Foi observada uma diferença significativa entre enseada e canal do rio em relação às variáveis transparência da água, que se apresentou maior na enseada, $\mathrm{e}$ velocidade da água, maior no canal. A diversidade de espécies também foi diferente entre os ambientes, com um valor significativamente maior na enseada. Nas duas localidades os maiores valores de riqueza e diversidade de espécies ocorreram durante o período de seca e os de densidade relativa (CPUE) durante a enchente. Em relação à abundância os ambientes foram mais semelhantes durante os períodos de cheia, quando também foi observada uma similaridade maior entre eles em relação às variáveis limnológicas. Isso ocorre devido à homogeneização da água e da estrutura dos hábitats em função da inundação. Os resultados indicam que o pulso de inundação, associado à velocidade da água, foi a principal força que influenciou a estrutura da ictiofauna nos ambientes amostrados.

PALAVRAS-CHAVE. Diversidade; limnologia; pulso de inundação; similaridade.

A geomorfologia é um dos fatores mais importantes que afeta a estrutura da comunidade de peixes em ambientes lóticos (Allan 1997, Gordon et al. 1995). Ela influencia essas comunidades à medida que vai gradualmente se alterando ao longo do canal do rio e, consequentemente, ocasionando mudanças nas características limnológicas dos ambientes, desde a região de cabeceira até a foz (VANNOTE et al. 1980).

Além das características geomorfológicas, as variações sazonais, causadas, principalmente, por flutuações na precipi- tação pluviométrica, também afetam a estrutura de comunidades de peixes na região de planície inundável (Lowe-MCConNeLL 1999). Essas variações alteram as características físicas e químicas da água, como $\mathrm{pH}$, condutividade, oxigênio dissolvido, fluxo e temperatura, o que é determinante na estruturação da ictiofauna (TejerINA-GARRo et al. 1998). Além disso, elas expandem e contraem sazonalmente os ambientes, regulando as comunidades aquáticas (Junk et al. 1989) e tornando estes ambientes muito dinâmicos e diversos em espécies.

Revista Brasileira de Zoologia 24 (2): 482-492, junho 2007 
Na planície de inundação do Rio das Mortes foram realizados poucos estudos sobre a ictiofauna (Melo \& RöPKe 2004, Melo et al. 2005) e dentre eles nenhum explora a variabilidade das comunidades entre diferentes tipos de hábitats. Considerando o dinamismo e a alta complexidade ambiental da planície de inundação deste rio, incluindo a heterogeneidade de hábitats no canal, trabalhos com este foco são importantes para avaliar a riqueza de espécies e a distribuição das comunidades de peixes nesta região. A relevância é maior ainda quando se considera o rápido crescimento da atividade agropecuária que vem transformando as paisagens e homogeneizando os ambientes.

Assim, o presente trabalho foi desenvolvido com a finalidade de definir a influência de variáveis limnológicas sobre a estrutura da ictiofauna em dois ambientes em condições geomorfológicas distintas, na planície de inundação do Rio das Mortes.

\section{MATERIAL E MÉTODOS}

\section{Área de estudo}

O Rio das Mortes é o principal afluente da margem esquerda do rio Araguaia, tanto pelo tamanho de sua área de drenagem, quanto pelo volume de água (Melo et al. 2005). Ele nasce na Serra de São Jerônimo, na Chapada dos Guimarães, Estado de Mato Grosso, e deságua no rio Araguaia, nas proximidades do município de São Félix do Araguaia, na Planície do Bananal, também em Mato Grosso (Fig. 1). Nesta região, o Rio das Mortes apresenta um canal muito sinuoso, com vários lagos de meandro, remansos e enseadas, e escoa sobre Plintossolos Distróficos Álicos ou Distróficos (BRASIL 1981). Drena áreas cobertas por vegetação de cerrado tipo savana aberta, interrompida em alguns pontos por pastagens (MELO \& RÖPKE 2004).

O clima da região é do tipo tropical continental sempre quente (MARIMON \& LiMA 2001), com dois períodos bem definidos: um úmido, entre dezembro e abril, e um de seca intensa, entre junho e setembro (DiEgues 2002). Os meses de maior precipitação pluviométrica são dezembro, janeiro e fevereiro. A temperatura média anual é de $24,9^{\circ} \mathrm{C}$ (MARIMON \& Lima 2001), sendo geralmente registradas em julho as menores temperaturas e em setembro as mais altas. No período de cheia, o Baixo Rio das Mortes conecta-se ao rio Araguaia, pela sua margem esquerda, através de cerca de $40 \mathrm{~km}$ de planície inundada, durante um período de, aproximadamente, quatro meses.

Neste estudo, as coletas foram realizadas em dois ambientes: em uma Enseada (E) no baixo Rio das Mortes e em um trecho do Canal deste rio (R). A Enseada está localizada na margem esquerda do Rio das Mortes (12 $2^{\circ} 31^{\prime} 45,0^{\prime \prime} \mathrm{S}$ e $50^{\circ} 59^{\prime}$ $\left.01,2^{\prime \prime} \mathrm{W}\right)$ e possui, aproximadamente, $600 \mathrm{~m}$ de comprimento, $100 \mathrm{~m}$ de largura e três metros de profundidade média. Neste ambiente foi amostrado um trecho de cerca de $100 \mathrm{~m}$. No Canal do Rio as coletas foram realizadas em um trecho de aproximadamente $100 \mathrm{~m}\left(12^{\circ} 31^{\prime} 17,1^{\prime \prime} \mathrm{S}\right.$ e $\left.50^{\circ} 58^{\prime} 45,1^{\prime \prime} \mathrm{W}\right)$, em sua margem direita. Este ponto, distante cerca de $1000 \mathrm{~m}$ da Enseada, possui cerca de $200 \mathrm{~m}$ de largura, durante o período de seca, e profundidade média de, aproximadamente, 3,5 m.

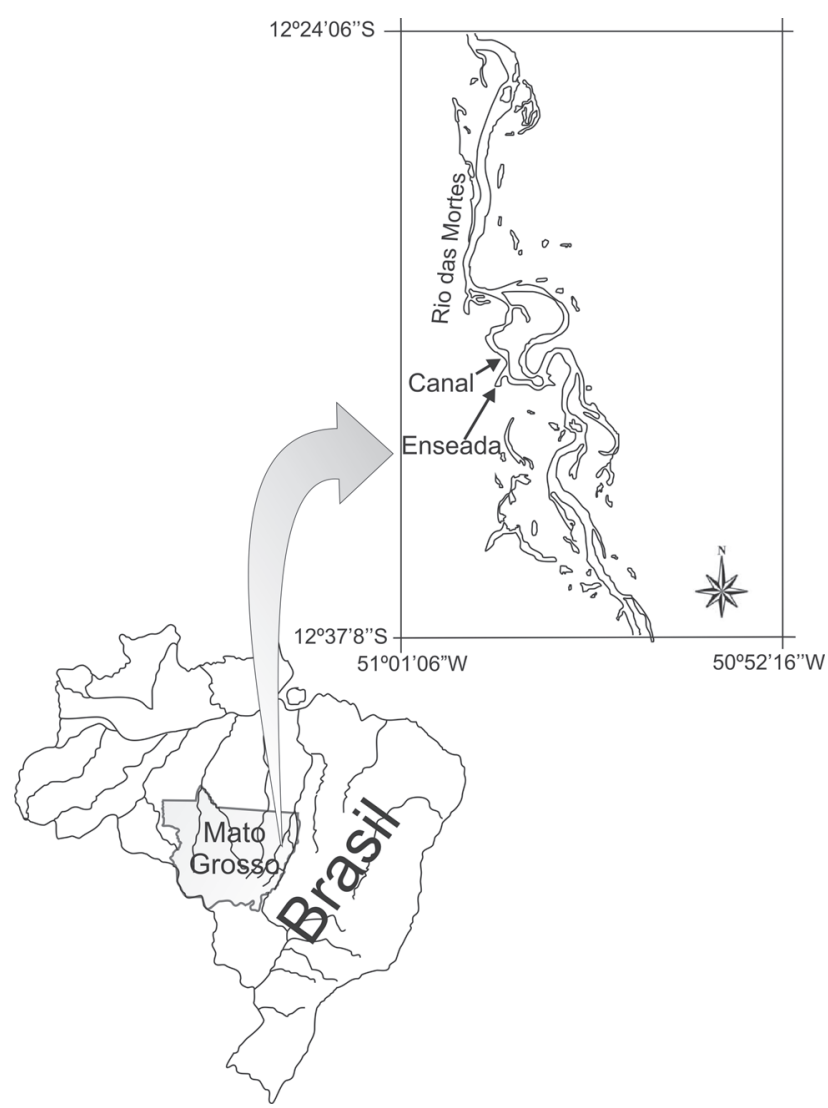

Figura 1. Mapa indicando a localização da enseada e do trecho amostrado no anal principal do Rio das Mortes Mato Grosso.

\section{Métodos}

As coletas foram realizadas em março (cheia), julho (vazante), outubro (seca) e dezembro (enchente), utilizando-se redes de emalhar de 30, 40, 50, 60, 70, 80, 100, 120, 140 e 180 mm entre nós opostos, com $10 \mathrm{~m}$ de comprimento e 1,70 m de altura. Em cada ambiente as redes ficaram expostas durante um período de 24 horas, sendo revisadas a cada quatro horas. Os peixes coletados foram fixados com solução de formalina 10\% e, posteriormente, triados e conservados em álcool 70\%. Todos os peixes coletados encontram-se depositados na coleção ictiológica do Laboratório de Ictiologia e Limnologia da Universidade do Estado de Mato Grosso, Campus de Nova Xavantina.

As variáveis limnológicas foram analisadas durante as quatro coletas na Enseada e no Canal do Rio. O oxigênio dissolvido foi analisado pelo método de Winkler (PINTO-SILVA 2002). Os valores de $\mathrm{pH}$ e condutividade elétrica foram obtidos com o uso de phmetro e condutivímetro QUIMIS, modelo Q-400 BC e modelo Q-405 B2, respectivamente. A temperatura da água foi medida com termômetro de mercúrio $\left(-10^{\circ} \mathrm{C}\right.$ a $\left.60^{\circ} \mathrm{C}\right)$. As amostras de água para estas análises foram retiradas da superfície e a $20 \mathrm{~cm}$ do fundo dos ambientes com o uso de garrafa de Van Dorn, a cada seis horas, durante as 24 horas de exposição 
das redes. A transparência da água foi obtida utilizando-se disco de Secchi (Esteves 1998).

A profundidade média foi obtida por medições realizadas próximo às baterias de redes e no meio do corpo de água, em cada um dos ambientes amostrados, com o uso de corda graduada. O material em suspensão foi quantificado por análise gravimétrica (PinTo-Silva 2002) em amostras coletadas na superfície da coluna de água. A velocidade da água foi medida próximo às baterias de rede, em cada ambiente, com o uso de fluxômetro mecânico General Oceanics.

Para analisar a similaridade entre os ambientes com base nas características limnológicas dos quatro períodos sazonais, os valores das variáveis limnológicas foram padronizados e, posteriormente, procedida a análise de agrupamento, com nível de corte em $80 \%$. Para esta análise utilizou-se o programa Statistica for Windows versão 6.0, método de ligação UPGMA (Agrupamento de Associação Média Não Ponderada) e distância Euclidiana na elaboração da matriz de similaridade. A comparação das variáveis limnológicas entre Enseada e Canal do Rio foi realizada com o uso do teste $t$ para observações independentes (ZAR 1996).

A diversidade de espécies $\left(\mathrm{H}^{\prime}\right)$, para o período anual e para os diferentes períodos sazonais em cada ambiente, foi determinada através do índice de Shannon-Wiener (MagurRan 2004): $H^{\prime}=-\Sigma\left(\right.$ pi. $\log _{2}$ pi), onde: pi = proporção de indivíduos encontrados em uma dada espécie.

A uniformidade foi calculada por meio da seguinte equação: $\mathrm{U}=\mathrm{H}^{\prime} / \mathrm{H}_{\max }$ onde: $\mathrm{U}=$ índice de uniformidade, $\mathrm{H}_{\max }=\log _{2}$ do número de espécies coletadas.

O teste $t$ para comparação de dois índices de diversidade (ZAR 1996) foi utilizado para testar diferenças entre a Enseada e o Canal do Rio, durante o período anual.

A abundância relativa das espécies, em dado período, foi determinada como o número de indivíduos de cada espécie dividido pelo total de indivíduos coletados no período. A densidade de peixes nos ambientes estudados foi obtida por meio da Captura por Unidade de Esforço (CPUE), calculada para o total de indivíduos coletados em cada período sazonal em $\mathrm{g} / \mathrm{m}^{2} / 24$ horas.

A similaridade entre os dois ambientes em relação à abundância foi determinada por meio do índice de Morisita-Horn (Krebs 1989). A matriz de similaridade obtida com esse índice foi utilizada para análise de agrupamento, com nível de corte em 55\%, utilizando-se o programa Statistica for Windows versão 6.0, método UPGMA (Agrupamento de Associação Média Não Ponderada).

\section{RESULTADOS}

\section{Variáveis limnológicas}

A análise de agrupamento com base nas variáveis limnológicas quantificadas mostrou a formação de três grupos principais (Fig. 2): o grupo 1, menos similar, inclui a Enseada e o Canal do Rio no mês de março (cheia). O grupo 2 inclui Ense-

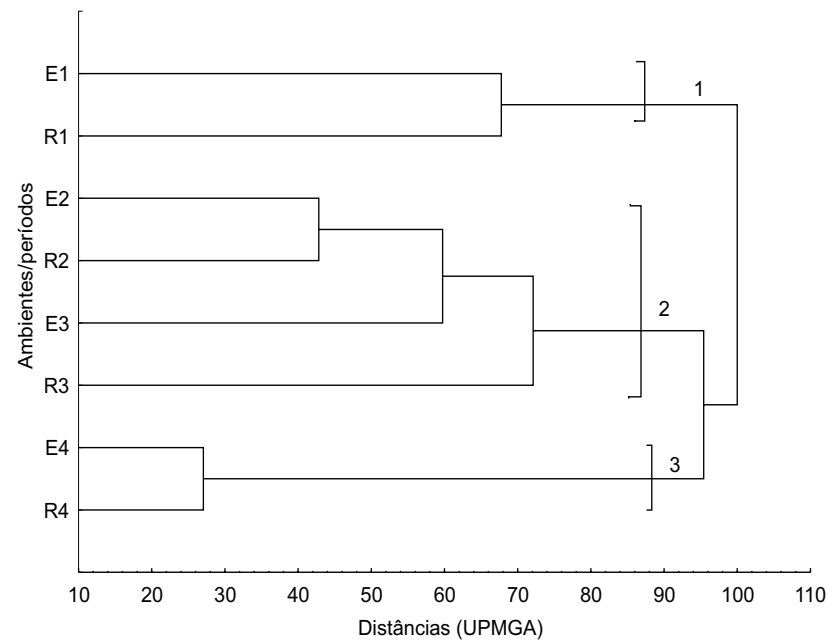

Figura 2. Agrupamento dos ambientes entre os quatro períodos de coleta com base nos dados padronizados das variáveis: $\mathrm{pH}$, condutividade, temperatura da água, oxigênio dissolvido, transparência, profundidade, velocidade da corrente e material em suspensão. Método de ligação UPGMA e matriz da distância Euclidiana. (E) Enseada, (R) canal do rio, (1) março, (2) julho, (3) outubro, (4) dezembro.

ada e Canal do Rio em julho (vazante), estando associados a este também a Enseada e o Canal do Rio em outubro (seca), e, por fim, o grupo 3 inclui a Enseada e o Canal do Rio em dezembro (enchente). Este último com maior similaridade entre os componentes. Estes agrupamentos indicam que, em relação às variáveis limnológicas, a similaridade foi maior entre um ambiente e outro em um mesmo período de coleta, principalmente nos períodos relacionados à época chuvosa, do que entre os diferentes períodos de análise em um mesmo ambiente.

De acordo com o teste $t$, os dois ambientes diferiram significativamente $(\mathrm{p}<0,05)$ apenas em relação às variáveis transparência $(\mathrm{t}=4,1 ; \mathrm{p}=0,0001)$ e velocidade da água $(\mathrm{t}=2,2 ; \mathrm{p}=$ $0,03)$. A média anual da velocidade da água no Canal do Rio $(15,4 \mathrm{~cm} / \mathrm{s})$ foi superior àquela observada na Enseada $(3,8 \mathrm{~cm} / \mathrm{s})$ (Tab. I). O inverso ocorreu em relação à transparência da água, que foi, em média, maior na Enseada $(101,5 \mathrm{~cm})$ do que no Canal do Rio $(70,4 \mathrm{~cm})$. Em relação às demais variáveis limnológicas não houve variação significativa entre os dois ambientes.

O pH da água teve médias anuais de 6,52 na Enseada e de 6,53 no Canal do Rio (Tab. I). Este parâmetro variou entre 6,32 e 6,82 na Enseada e entre 6,18 e 6,81 no Canal. A concentração média de oxigênio dissolvido na água foi de $6,65 \mathrm{mg} / \mathrm{l}$ no Canal do Rio (4,90 mg/l a 8,13 mg/l) e de $6,10 \mathrm{mg} / \mathrm{l}$ na Enseada $(4,10$ $\mathrm{mg} / \mathrm{l}$ a $7,93 \mathrm{mg} / \mathrm{l})$. A condutividade da água foi baixa ao longo de todo o ano nos dois ambientes, sendo as médias anuais iguais a $10,51 \mu \mathrm{Scm}^{-1}$ na Enseada (mínimo $=9,39 \mu \mathrm{Scm}^{-1}$; máximo $=$ $\left.11,38 \mu \mathrm{Scm}^{-1}\right)$ e $11,05 \mu \mathrm{Scm}^{-1}$ no Canal do Rio $\left(9,53 \mu \mathrm{Scm}^{-1}\right.$ a $\left.11,95 \mu \mathrm{Scm}^{-1}\right)$. (Tab. I). 
Tabela I. Valores médios (em negrito) e coeficientes de variação (entre parênteses) das variáveis limnológicas analisadas em março (1), julho (2), outubro (3) e dezembro (4) e no período anual na enseada (E) a no canal do rio (R). Os ambientes diferiram significativamente $(0,05)$ apenas em relação às variáveis transparência da água e velocidade da água.

\begin{tabular}{|c|c|c|c|c|c|c|c|c|}
\hline \multirow[b]{2}{*}{ Períodos } & \multicolumn{8}{|c|}{ Variáveis limnológicas } \\
\hline & $\mathrm{pH}$ & $\begin{array}{l}\text { Condutividade } \\
\qquad(\mu \mathrm{S} \mathrm{cm}-1)\end{array}$ & $\begin{array}{c}\text { Temperatura } \\
\left({ }^{\circ} \mathrm{C}\right)\end{array}$ & $\begin{array}{c}\text { Oxigênio } \\
\text { dissolvido }(\mathrm{mg} / \mathrm{l})\end{array}$ & $\begin{array}{l}\text { Transparência } \\
(\mathrm{cm})\end{array}$ & $\begin{array}{l}\text { Profundidade } \\
(\mathrm{m})\end{array}$ & $\begin{array}{l}\text { Velocidade da } \\
\text { água }(\mathrm{cm} / \mathrm{s})\end{array}$ & $\begin{array}{c}\text { Matéria em } \\
\text { Suspensão (mg/l) }\end{array}$ \\
\hline E1 & $6,32(2,82)$ & $11,38(4,74)$ & $29,51(3,68)$ & $4,10(12,61)$ & $130,5(4,13)$ & $5,00(31,60)$ & $15,17(130,34)$ & 0,006 \\
\hline E2 & $6,51(1,63)$ & $11,06(5,70)$ & $27,10(5,69)$ & $7,93(2,21)$ & $106,6(15,02)$ & $1,65(54,17)$ & $0,00(0,00)$ & 0,004 \\
\hline E3 & $6,82(1,61)$ & $10,12(2,96)$ & $30,23(2,45)$ & $6,78(6,54)$ & $105,5(5,77)$ & $1,88(48,90)$ & $0,00(0,00)$ & 0,003 \\
\hline E4 & $6,53(3,11)$ & $9,39(4,00)$ & $26,38(2,82)$ & $6,22(5,80)$ & $63,5(10,05)$ & $2,92(23,15)$ & $0,00(0,00)$ & 0,009 \\
\hline R1 & $6,18(1,73)$ & $11,95(3,82)$ & $29,08(2,56)$ & $4,90(5,52)$ & $80,5(14,57)$ & $4,96(7,05)$ & $3,21(172,76)$ & 0,009 \\
\hline R2 & $6,81(1,27)$ & $11,93(9,56)$ & $27,22(5,45)$ & $8,13(1,85)$ & $89,8(8,40)$ & $2,37(23,05)$ & $26,75(68,38)$ & 0,007 \\
\hline R3 & $6,78(2,72)$ & $11,00(1,89)$ & $29,84(2,97)$ & $6,87(5.19)$ & $83,5(5,12)$ & $2,63(22,97)$ & $26,42(136,16)$ & 0,017 \\
\hline R4 & $6,42(6,42)$ & $9,53(7,07)$ & $25,91(2,62)$ & $7,06(4,37)$ & $28,0(5,05)$ & $3,74(17,17)$ & $5,37(242,95)$ & 0,013 \\
\hline E Anual & $6,52(3,48)$ & $10,51(8,95)$ & $28,18(6,80)$ & $6,10(24,22)$ & $101,5(25,80)$ & $3,03(58,57)$ & $3,79(300,65)$ & $0,006(48,10)$ \\
\hline R Anual & $6,53(5,30)$ & $11,05(10,79)$ & $28,07(6,57)$ & $6,65(18,00)$ & $70,4(37,16)$ & $3,42(34,09)$ & $15,44(148,83)$ & $0,011(38,56)$ \\
\hline
\end{tabular}

\section{Estrutura da ictiofauna}

Durante o período de estudo foram capturados 2000 peixes, distribuídos em 80 espécies, 19 famílias e cinco ordens. Characiformes foi a ordem dominante durante o período anual na Enseada (70\%) e no Canal do Rio (71\%), seguida por Siluriformes (23\% e 24\%, respectivamente) (Fig. 3). Gymnotiformes ocorreu apenas na Enseada e foi a ordem menos abundante ao longo do ano neste ambiente $(0,1 \%)$ enquanto no Canal do Rio, Perciformes foi a ordem com abundância relativa mais baixa durante todo o ano de amostragem $(0,6 \%)$.

Characidae foi a família mais abundante tanto na Enseada, quanto no Canal do Rio, representando 33\% e 27\%, respectivamente, do total de indivíduos capturados para cada um dos ambientes (Fig. 4). Auchenipteridae destacou-se como a segunda família com maior abundância relativa nos dois ambientes, com 19\% dos indivíduos coletados na Enseada e 22\% no Canal do Rio. A família menos abundante na Enseada foi Sternopygidae, com apenas um indivíduo, e no Canal do Rio foi Loricariidae, com seis indivíduos.

Do total de indivíduos coletados, para a Enseada foi obtida a maior abundância (1061) em relação ao trecho amostrado no Canal do Rio (939) (Tab. II). Na Enseada também foram observados valores mais altos de riqueza de espécies (77), de famílias (19) e de ordens (5) do que os encontrados para o Canal do Rio (43 espécies, 15 famílias e quatro ordens). Psectrogaster amazonica Eigenmann \& Eigenmann, 1889 foi a espécie dominante na Enseada, com 63 indivíduos, o que corresponde a cerca de $6 \%$ do total de espécimes capturados ao longo do ano neste ambiente. No Canal do Rio Triportheus auritus (Valenciennes, 1850) foi a espécie dominante durante o período anual, com 86 espécimes, que representaram $9 \%$ do total capturado para este ambiente.

Os dois ambientes estudados apresentaram altos valores de diversidade de espécies ao longo do ano (Tab. III), no entanto

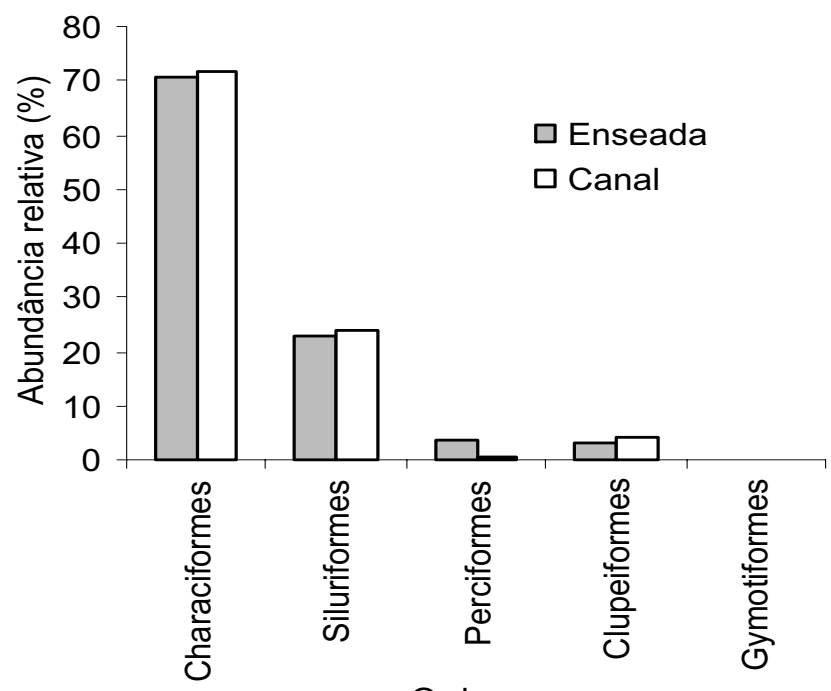

Figura 3. Abundância relativa das ordens de Osteichthyes coletadas durante o período anual na enseada e no canal principal do Rio das Mortes.

o valor encontrado na Enseada (5,49 bits/indivíduo) foi maior do que aquele observado no Canal do Rio (4,44 bits/ indivíduo), assim como a riqueza de espécies (77 espécies na Enseada e 43 no Canal do Rio). A uniformidade foi alta para os dois ambientes, sendo igual a 0,87 na Enseada e 0,81 no Canal do Rio.

$\mathrm{Na}$ análise sazonal a maior diversidade de espécies foi encontrada no mês de outubro (seca), tanto na Enseada (5,09 bits/ indivíduo), quanto no Canal do Rio (4,0 bits/ indivíduo) (Tab. III). Nesse período também foi coletado o maior número de espécies em ambos os ambientes (52 espécies na enseada e 31 no

Revista Brasileira de Zoologia 24 (2): 482-492, junho 2007 


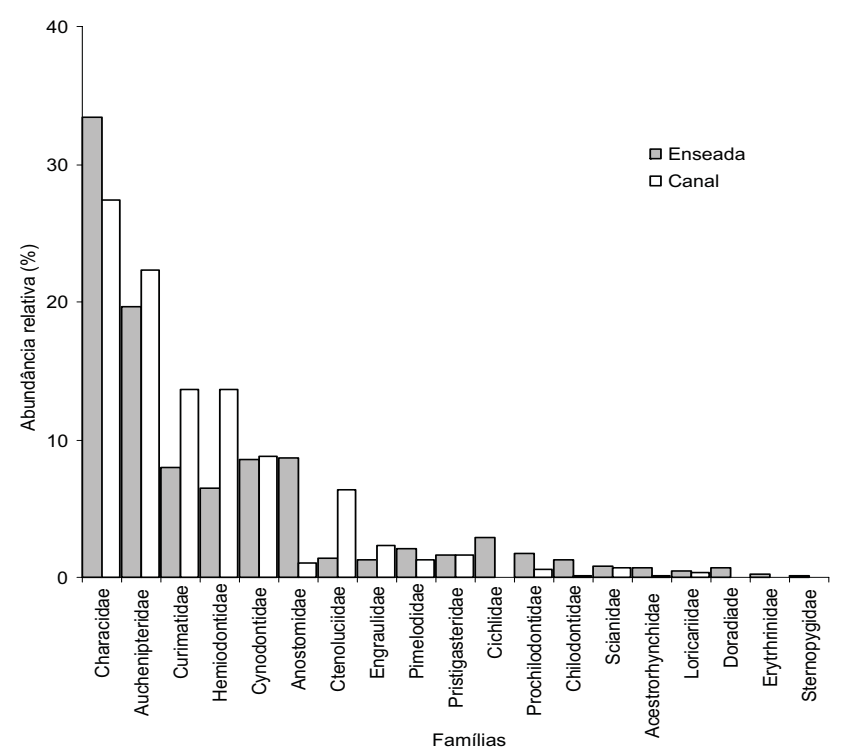

Figura 4. Abundância relativa das famílias de Osteichthyes coletadas ao longo do ano na enseada e no canal principal do Rio das Mortes. canal do rio). Os menores valores de diversidade e riqueza de espécies ocorreram em março nos dois ambientes (Tab. III). Em relação à uniformidade, os maiores valores na Enseada ocorreram nos meses de julho e outubro, que correspondem ao período de seca, enquanto que, no Canal do Rio, estes foram registrados no mês de março, período correspondente ao pico de cheia.

Os valores de densidade de peixes em biomassa, medidos pela captura por unidade de esforço (CPUE), foram muito próximos no período anual entre Enseada e Canal do Rio (Tab. III). Na análise sazonal o maior valor ocorreu em dezembro nos dois ambientes. Os valores de dominância de espécies foram considerados baixos nos ambientes estudados ao longo de todos os períodos, como mostram os dados da tabela III e as curvas de dominância e seus respectivos índices de uniformidades nas figuras 5 e 6 .

A análise de agrupamento com base nos dados de abundância mostrou a ocorrência de três grupos mais consistentes (Fig. 7). O grupo 1 é formado pelos peixes coletados em março na Enseada e no Canal do Rio e pelos peixes coletados em julho no Canal do Rio. O grupo 2 inclui os peixes coletados em dezembro nos dois ambientes e o grupo 3 os peixes coletados em julho e outubro na Enseada. Esses agrupamentos indicam a

Tabela II. Número de indivíduos coletados nas espécies e taxa superiores na enseada e no trecho amostrado do canal principal do Rio das Mortes, em cada período de coleta e no período anual. A ordem taxonômica está de acordo com ReIs et al. (2003). (M) Março, (J) julho, (O) outubro, (D) dezembro.

\begin{tabular}{|c|c|c|c|c|c|c|c|c|c|}
\hline \multirow{3}{*}{ Espécies } & \multicolumn{9}{|c|}{ Número de indivíduos } \\
\hline & \multicolumn{4}{|c|}{ Enseada } & \multicolumn{5}{|c|}{ Canal } \\
\hline & M & J & $\mathrm{O}$ & $\mathrm{D}$ & M & J & $\mathrm{O}$ & $\mathrm{D}$ & Anual \\
\hline Clupeiformes & 13 & 4 & 0 & 31 & 4 & 10 & 11 & 27 & 100 \\
\hline \multicolumn{10}{|l|}{ Engraulidae } \\
\hline Lycengraulis batesii (Günther, 1868) & 1 & 2 & 0 & 11 & 0 & 4 & 5 & 13 & 36 \\
\hline Pristigasteridae & 6 & 1 & 0 & 10 & 2 & 3 & 3 & 7 & 32 \\
\hline Pellona castelnaeana Valenciennes, 1847 & 4 & 0 & 0 & 8 & 0 & 3 & 1 & 5 & 21 \\
\hline Pristigaster cayana Cuvier, 1829 & 2 & 1 & 0 & 2 & 2 & 0 & 2 & 2 & 11 \\
\hline Characiformes & 61 & 216 & 173 & 297 & 22 & 102 & 223 & 325 & 1419 \\
\hline \multicolumn{10}{|l|}{ Curimatidae } \\
\hline Curimata cyprinoides (Linnaeus, 1766) & 0 & 2 & 1 & 5 & 0 & 0 & 39 & 6 & 53 \\
\hline Curimatella dorsalis (Eigenmann \& Eigenmann, 1889) & 0 & 0 & 10 & 0 & 0 & 0 & 0 & 0 & 10 \\
\hline Cyphocarax spiluropsis (Eigenmann \& Eigenmann, 1889) & 0 & 1 & 0 & 0 & 0 & 0 & 0 & 0 & 1 \\
\hline Cyphocarax stilbolepis Vari, 1992 & 0 & 2 & 1 & 0 & 0 & 0 & 0 & 0 & 3 \\
\hline Psectrogaster amazonica Eigenmann \& Eigenmann, 1889 & 0 & 24 & 15 & 24 & 0 & 0 & 0 & 83 & 146 \\
\hline \multicolumn{10}{|l|}{ Prochilodontidae } \\
\hline Prochilodus nigricans Agassiz, 1829 & 0 & 5 & 2 & 1 & 0 & 0 & 0 & 0 & 8 \\
\hline Semaprochilodus brama (Valenciennes 1850) & 1 & 4 & 3 & 3 & 0 & 0 & 0 & 5 & 16 \\
\hline \multicolumn{10}{|l|}{ Anostomidae } \\
\hline Anostomoides laticeps (Eigenmann, 1912) & 0 & 0 & 0 & 1 & 0 & 0 & 0 & 0 & 1 \\
\hline Laemolyta fernandezi Myers, 1950 & 0 & 20 & 11 & 1 & 0 & 1 & 0 & 0 & 33 \\
\hline Leporinus affinis Günther, 1864 & 0 & 6 & 1 & 2 & 0 & 4 & 4 & 0 & 17 \\
\hline
\end{tabular}




\begin{tabular}{|c|c|c|c|c|c|c|c|c|c|}
\hline \multirow{3}{*}{ Espécies } & \multicolumn{9}{|c|}{ Número de indivíduos } \\
\hline & \multicolumn{4}{|c|}{ Enseada } & \multicolumn{5}{|c|}{ Canal } \\
\hline & M & J & $\mathrm{O}$ & $\mathrm{D}$ & M & $\mathrm{J}$ & $\mathrm{O}$ & $\mathrm{D}$ & Anual \\
\hline Leporinus friderici (Bloch, 1794) & 0 & 11 & 4 & 10 & 0 & 0 & 0 & 0 & 25 \\
\hline Leporinus sp.1 & 0 & 3 & 0 & 0 & 0 & 0 & 0 & 0 & 3 \\
\hline Leporinus sp.2 & 0 & 0 & 1 & 0 & 0 & 0 & 0 & 0 & 1 \\
\hline Leporinus trifasciatus Steindachner, 1876 & 1 & 0 & 1 & 0 & 0 & 0 & 0 & 0 & 2 \\
\hline Schizodon vittatus (Valenciennes, 1850) & 0 & 8 & 2 & 9 & 0 & 0 & 0 & 1 & 20 \\
\hline \multicolumn{10}{|l|}{ Chilodontidae } \\
\hline Caenotropus labyrinticus (Kner, 1858) & 0 & 8 & 6 & 0 & 0 & 1 & 0 & 0 & 15 \\
\hline \multicolumn{10}{|l|}{ Hemiodontidae } \\
\hline Anodus orinocensis (Steindachner, 1887) & 0 & 0 & 2 & 48 & 0 & 5 & 39 & 33 & 127 \\
\hline Argonectes robertsi Langeani, 1999 & 0 & 0 & 0 & 1 & 0 & 0 & 0 & 0 & 1 \\
\hline Hemiodus argenteus Pellegrin, 1908 & 0 & 3 & 2 & 0 & 0 & 0 & 24 & 0 & 29 \\
\hline Hemiodus microlepis Kner, 1858 & 0 & 0 & 0 & 0 & 0 & 0 & 12 & 0 & 12 \\
\hline Hemiodus unimaculatus (Bloch, 1794) & 0 & 0 & 12 & 1 & 0 & 0 & 14 & 1 & 28 \\
\hline \multicolumn{10}{|l|}{ Characidae } \\
\hline Agoniates halecinus Muller \& Troschel, 1845 & 15 & 2 & 0 & 19 & 2 & 6 & 4 & 0 & 48 \\
\hline Brycon falcatus Muler \& Troschel, 1844 & 0 & 5 & 0 & 3 & 0 & 0 & 0 & 0 & 8 \\
\hline Brycon pesu Muller \& Troschel, 1845 & 0 & 0 & 0 & 0 & 0 & 1 & 2 & 0 & 3 \\
\hline Bryconops alburnoides Kner, 1858 & 9 & 1 & 4 & 20 & 0 & 2 & 11 & 1 & 48 \\
\hline Chalceus epakros Zanata \& Toledo-Piza, 2004 & 0 & 16 & 2 & 0 & 0 & 0 & 4 & 2 & 24 \\
\hline Metynnis hypsauchen Muller \& Troschel, 1844 & 0 & 10 & 4 & 9 & 0 & 0 & 0 & 0 & 23 \\
\hline Metynnis lippincottianus (Cope,1870) & 0 & 1 & 0 & 0 & 0 & 0 & 0 & 0 & 1 \\
\hline Myleus schomburgkii (Jardine \& Schomburgk, 1841) & 0 & 2 & 6 & 8 & 0 & 0 & 0 & 0 & 16 \\
\hline Myleus torquatus (Kner,1858) & 0 & 11 & 3 & 3 & 0 & 0 & 2 & 1 & 20 \\
\hline Myleus sp. 1 & 0 & 0 & 0 & 4 & 0 & 0 & 0 & 0 & 4 \\
\hline Myleus asterias (Muller \& Troschel, 1844) & 0 & 0 & 0 & 7 & 0 & 0 & 0 & 0 & 7 \\
\hline Pygocentrus nattereri Kner, 1858 & 1 & 12 & 3 & 9 & 2 & 3 & 1 & 0 & 31 \\
\hline Roeboides sp. & 0 & 1 & 1 & 0 & 0 & 0 & 0 & 0 & 2 \\
\hline Serrasalmus eigenmanni Norman, 1929 & 3 & 10 & 12 & 15 & 0 & 13 & 3 & 0 & 56 \\
\hline Serrasalmus geryi Jégu \& Santos, 1988 & 1 & 2 & 1 & 0 & 0 & 0 & 2 & 0 & 6 \\
\hline Serrasalmus rhombeus (Linnaeus, 1766) & 1 & 14 & 6 & 2 & 4 & 8 & 0 & 0 & 35 \\
\hline Tetragonopterus argenteus Cuvier, 1816 & 0 & 0 & 4 & 5 & 0 & 0 & 0 & 0 & 9 \\
\hline Tetragonopterus chalceus Spix \& Agassiz, 1829 & 0 & 1 & 0 & 0 & 0 & 0 & 0 & 0 & 1 \\
\hline Tetragonopterus sp. & 0 & 2 & 0 & 0 & 0 & 0 & 0 & 0 & 2 \\
\hline Triportheus albus Cope, 1872 & 1 & 9 & 6 & 5 & 0 & 3 & 5 & 6 & 35 \\
\hline Triportheus auritus (Valenciennes, 1850) & 4 & 0 & 2 & 25 & 3 & 10 & 10 & 63 & 117 \\
\hline Triportheus trifurcatus (Castelnau, 1890) & 0 & 5 & 11 & 15 & 1 & 3 & 9 & 70 & 114 \\
\hline \multicolumn{10}{|l|}{ Acestrorhynchidae } \\
\hline Acestrorhynchus falcirostris (Cuvier, 1819) & 0 & 1 & 1 & 3 & 0 & 0 & 0 & 0 & 5 \\
\hline Acestrorhynchus microlepis (Schomburgk, 1841 ) & 0 & 0 & 2 & 0 & 0 & 0 & 1 & 0 & 3 \\
\hline \multicolumn{10}{|l|}{ Cynodontidae } \\
\hline Cynodon gibbus Spix \& Agassiz, 1829 & 0 & 1 & 0 & 0 & 0 & 0 & 0 & 1 & 2 \\
\hline Hydrolycus armatus (Jardine \& Schomburgk, 1841) & 1 & 1 & 1 & 0 & 0 & 0 & 1 & 2 & 6 \\
\hline
\end{tabular}


Tabela II. Continuação.

\begin{tabular}{|c|c|c|c|c|c|c|c|c|c|}
\hline \multirow{3}{*}{ Espécies } & \multicolumn{9}{|c|}{ Número de indivíduos } \\
\hline & \multicolumn{4}{|c|}{ Enseada } & \multicolumn{5}{|c|}{ Canal } \\
\hline & M & J & $\mathrm{O}$ & $\mathrm{D}$ & $\mathrm{M}$ & 1 & $\mathrm{O}$ & D & Anual \\
\hline Hydrolycus tatauaia Toledo-Piza et al. 1999 & 3 & 8 & 26 & 7 & 0 & 0 & 2 & 7 & 53 \\
\hline Rhaphiodon vulpinus Spix \& Agassiz, 1829 & 15 & 0 & 1 & 27 & 10 & 18 & 2 & 39 & 112 \\
\hline \multicolumn{10}{|l|}{ Erythrinidae } \\
\hline Hoplias malabaricus (Bloch, 1794) & 0 & 1 & 1 & 0 & 0 & 0 & 0 & 0 & 2 \\
\hline \multicolumn{10}{|l|}{ Ctenoluciidae } \\
\hline Boulengerella cuvieri (Agassiz, 1829) & 5 & 3 & 2 & 5 & 0 & 24 & 32 & 4 & 75 \\
\hline Siluriformes & 52 & 31 & 20 & 140 & 11 & 25 & 16 & 172 & 467 \\
\hline \multicolumn{10}{|l|}{ Loricariidae } \\
\hline Hypoptopoma sp. & 0 & 0 & 2 & 2 & 0 & 0 & 0 & 3 & 7 \\
\hline Hypostomus sp. & 0 & 0 & 0 & 1 & 0 & 0 & 0 & 0 & 1 \\
\hline \multicolumn{10}{|l|}{ Pimelodidae } \\
\hline Pimelodus blochii Valenciennes, 1840 & 0 & 6 & 5 & 0 & 0 & 4 & 1 & 0 & 16 \\
\hline Pinirampus pirinampu (Spix \& Agassiz, 1829) & 0 & 0 & 0 & 0 & 1 & 0 & 0 & 1 & 2 \\
\hline Pseudoplatystoma fasciatum (Linnaeus, 1766) & 0 & 0 & 0 & 2 & 0 & 0 & 0 & 0 & 2 \\
\hline Sorubim lima (Bloch \& Schneider, 1801) & 0 & 5 & 2 & 2 & 0 & 0 & 1 & 4 & 14 \\
\hline \multicolumn{10}{|l|}{ Doradidae } \\
\hline Hassar wilderi Kindle, 1895 & 0 & 3 & 4 & 0 & 0 & 0 & 0 & 0 & 7 \\
\hline \multicolumn{10}{|l|}{ Auchenipteridae } \\
\hline Ageneiosus inermis (Linnaeus, 1766) & 22 & 2 & 3 & 28 & 3 & 2 & 9 & 57 & 126 \\
\hline Ageneiosus sp. & 3 & 0 & 0 & 10 & 1 & 0 & 1 & 43 & 58 \\
\hline Auchenipterichthys coracoideus (Eigenmann \& Allen, 1942) & 8 & 13 & 2 & 33 & 2 & 10 & 1 & 1 & 70 \\
\hline Auchenipterichthys longimanus (Günther, 1864) & 0 & 0 & 0 & 13 & 0 & 0 & 0 & 2 & 15 \\
\hline Auchenipterus nuchali (Spix \& Agassiz, 1829) & 18 & 2 & 2 & 21 & 4 & 9 & 3 & 60 & 119 \\
\hline Tatia sp. 1 & 0 & 0 & 0 & 1 & 0 & 0 & 0 & 0 & 1 \\
\hline Tatia sp. 2 & 0 & 0 & 0 & 2 & 0 & 0 & 0 & 0 & 2 \\
\hline Trachelyopterus galeatus (Linnaeus, 1766) & 1 & 0 & 0 & 25 & 0 & 0 & 0 & 1 & 27 \\
\hline Gymnotiformes & 0 & 0 & 0 & 1 & 0 & 0 & 0 & 0 & 1 \\
\hline \multicolumn{10}{|l|}{ Sternopygidae } \\
\hline Sternopygus obtusirostris Steindachner, 1881 & 0 & 0 & 0 & 1 & 0 & 0 & 0 & 0 & 1 \\
\hline Perciformes & 0 & 15 & 22 & 2 & 0 & 1 & 0 & 5 & 45 \\
\hline \multicolumn{10}{|l|}{ Sciaenidae } \\
\hline Pachyurus schomburgkii Günther, 1860 & 0 & 0 & 4 & 1 & 0 & 0 & 0 & 0 & 5 \\
\hline Plagioscion squamosissimus (Heckel, 1840) & 0 & 1 & 1 & 1 & 0 & 1 & 0 & 5 & 9 \\
\hline \multicolumn{10}{|l|}{ Cichlidae } \\
\hline Aequidens pallidus (Heckel, 1840) & 0 & 1 & 0 & 0 & 0 & 0 & 0 & 0 & 1 \\
\hline Biotodoma sp. Eigenmann \& Kennedy, 1903 & 0 & 3 & 5 & 0 & 0 & 0 & 0 & 0 & 8 \\
\hline Cichla temensis Humboldt, 1821 & 0 & 0 & 1 & 0 & 0 & 0 & 0 & 0 & 1 \\
\hline Crenicichla cf. johanna Heckel, 1840 & 0 & 0 & 1 & 0 & 0 & 0 & 0 & 0 & 1 \\
\hline Crenicichla cf. lugubris Heckel, 1840 & 0 & 1 & 1 & 0 & 0 & 0 & 0 & 0 & 2 \\
\hline Geophagus surinamensis (Bloch, 1791) & 0 & 7 & 5 & 0 & 0 & 0 & 0 & 0 & 12 \\
\hline Mesonauta acora (Castelnau, 1855) & 0 & 2 & 0 & 0 & 0 & 0 & 0 & 0 & 2 \\
\hline Satanoperca pappaterra (Heckel, 1840) & 0 & 0 & 4 & 0 & 0 & 0 & 0 & 0 & 4 \\
\hline Anual & 120 & 265 & 215 & 461 & 35 & 135 & 247 & 522 & 2000 \\
\hline
\end{tabular}

Revista Brasileira de Zoologia 24 (2): 482-492, junho 2007 

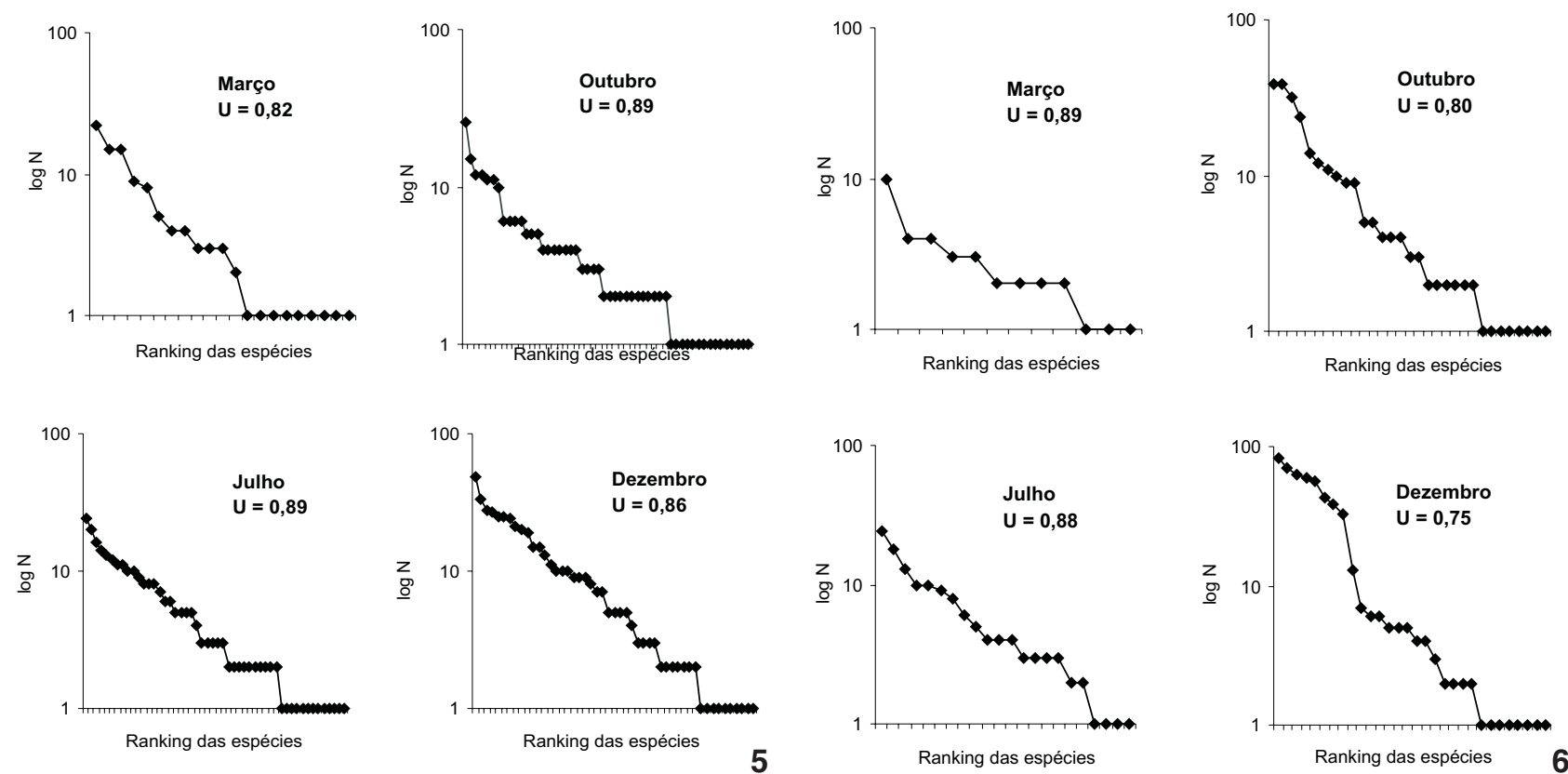

Figura 5. Curvas de dominância e uniformidades das comunidades de peixes da enseada (5) e do canal do rio (6) nos meses de março, julho, outubro e dezembro.

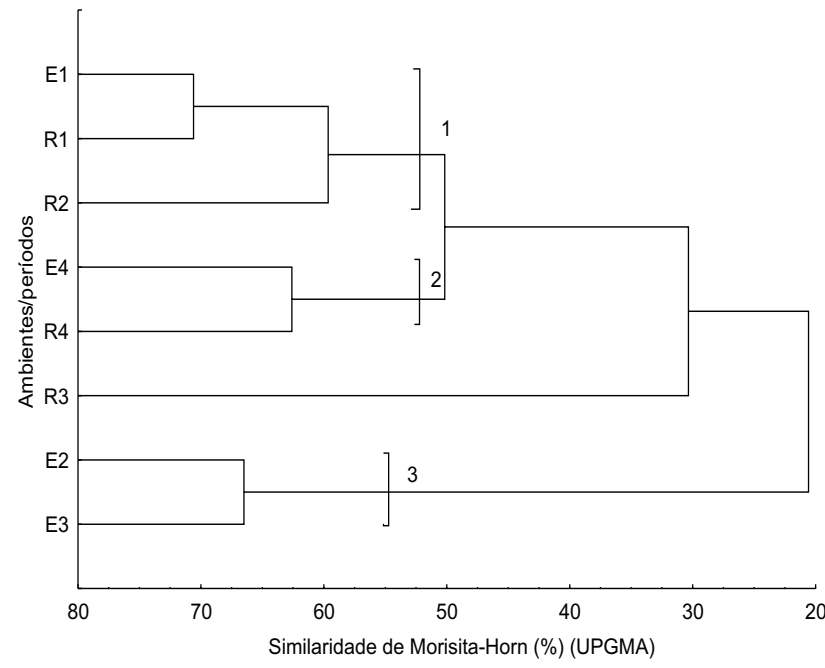

Figura 7. Agrupamento dos ambientes em relação à abundância de peixes entre os quatro períodos amostrados. (E) Enseada, (R) canal do rio, (1) março, (2) julho, (3) outubro, (4) dezembro.

influência da sazonalidade sobre os ambientes, tornando-os mais similares nos meses relacionados ao período de cheia.

\section{DISCUSSÃO}

\section{Variáveis limnológicas}

Os estudos limnológicos são de grande importância para a compreensão da estruturação da ictiofauna em ambientes
Tabela III. Riqueza (S), diversidade (H'), uniformidade (U), e densidade em biomassa (CPUE) das assembléias de peixes da enseada e do canal do rio durante os quatro períodos de coleta e no período anual.

\begin{tabular}{|c|c|c|c|c|c|c|c|c|}
\hline \multirow{3}{*}{ Período } & \multicolumn{8}{|c|}{ Local } \\
\hline & \multicolumn{4}{|c|}{ Enseada } & \multicolumn{4}{|c|}{ Canal do rio } \\
\hline & $S$ & $\mathrm{H}^{\prime}$ & U & CPUE & $S$ & $\mathrm{H}^{\prime}$ & $U$ & CPUE \\
\hline Março & 22 & 3,68 & 0,82 & 64,6 & 12 & 3,22 & 0,89 & 14,6 \\
\hline Julho & 50 & 5,07 & 0,89 & 54,8 & 22 & 3,96 & 0,88 & 64,9 \\
\hline Outubro & 52 & 5,09 & 0,89 & 44,3 & 31 & 4,00 & 0,80 & 71,2 \\
\hline Dezembro & 49 & 4,88 & 0,86 & 163,7 & 30 & 3,68 & 0,75 & 170,2 \\
\hline Anual & 77 & 5,497 & 0,87 & 81,8 & 43 & 4,44 & 0,81 & 80,2 \\
\hline
\end{tabular}

lóticos e lênticos (Tejerina-Garro 1998, Lowe-McConnell 1999). $\mathrm{O}$ pH e o oxigênio dissolvido na água são, freqüentemente, relacionados às alterações sazonais na estrutura da comunidade e na distribuição das espécies de peixes em planícies inundáveis tropicais (Junk et al. 1983, GouldiNG et al. 1988).

$\mathrm{Na}$ maioria dos ambientes aquáticos continentais o $\mathrm{pH}$ varia entre 6 e 8 (Esteves 1998). Dessa forma, os valores observados na Enseada e no Canal do Rio são considerados normais. Nestes ambientes, o pH foi variável ao longo do ano, apresentando valores mais baixos no período de cheia, quando o nível da água sobe e aumenta o processo de decomposição, o que, segundo Esteves (1998), provoca um declínio no $\mathrm{pH}$.

A quantidade de oxigênio dissolvido na água também é uma característica limnológica muito importante na dinâmica 
e caracterização dos ecossistemas aquáticos (WETZEL 1993, EsTeves 1998). Embora a concentração de oxigênio dissolvido apresente alta variabilidade entre diferentes localidades dentro de um rio (BAYLEy 1995), ela não diferiu entre a Enseada e o Canal do Rio das Mortes. Por outro lado, essa concentração foi variável entre períodos de amostragem, sofrendo um declínio durante o período de cheia nestes dois ambientes. Isso também é conseqüência do aumento do processo de decomposição na coluna de água (BAyley 1995).

Os baixos valores de condutividade elétrica da água na Enseada e no Canal do Rio indicam pequena quantidade de nutrientes, o que é comum em corpos de água do Brasil Central, onde os solos são pobres do ponto de vista geoquímico (Sioli 1991). De acordo com Lowe-McConnell (1999), nestes ambientes de águas claras, a condutividade varia entre 6 e $50 \mu \mathrm{S} \mathrm{cm}^{-1}$. Entretanto, apesar da pobreza em nutrientes, esses ambientes são capazes de sustentar uma rica ictiofauna (Melo et al. 2003).

\section{Estrutura da ictiofauna}

O curso inferior do Rio das Mortes é bastante sinuoso, com uma ampla área de inundação lateral, características geralmente associadas à alta diversidade de espécies (Junk 1980, AgostinHo et al. 1997). Em áreas inundáveis, como a do Rio das Mortes, a inundação da vegetação marginal durante o período chuvoso aumenta a disponibilidade de microhabitats, onde muitas espécies de peixes encontram recursos alimentares, abrigo e condições de reprodução, o que aumenta a riqueza e a diversidade local (Goulding 1980, Junk 1980, 1993, Melo et al. 2003).

As características geomorfológicas e hidrológicas da região inferior do Rio das Mortes fazem dela um local de grande diversidade de espécies, como indicam os altos índices de diversidade encontrados na Enseada $\left(\mathrm{H}^{\prime}=5,49\right)$ e no Canal do Rio $\left(\mathrm{H}^{\prime}=\right.$ $4,44)$. Estes índices são similares àqueles observados em outros rios amazônicos de planície de inundação, como Rio Negro $\left(\mathrm{H}^{\prime}\right.$ entre 2,46 e 4,70) (Goulding et al. 1988), Rio Juruá $\left(H^{\prime}=5,33\right)$ (Silvano et al. 2000) e o Tocantins $\left(\mathrm{H}^{\prime}=4,446\right)$ (Merona 1986).

Os altos índices de diversidade observados nos dois ambientes estudados estão relacionados aos elevados valores de uniformidade de espécies, os quais demonstram baixa dominância na comunidade (MAgURRAN 2004). Essa também é uma característica das comunidades de peixes da Bacia Amazônica, onde somente em ambientes alterados ou em momentos de migrações são observadas espécies com dominância maior que 45\% (SANTOS \& Ferreira 1999). Na Enseada e no Canal do Rio as espécies mais abundantes no período anual (Psectrogaster amazonica e Triportheus auritus, respectivamente) representaram menos de $10 \%$ cada uma do total de indivíduos capturados nestes ambientes.

Uma outra característica dos ambientes aquáticos amazônicos é a ocorrência de uma menor quantidade de espécies abundantes em relação às raras (Goulding et al. 1988, SiqueiraSouza \& Freitas 2004). Isso também foi observado na Enseada e no Canal do Rio das Mortes, onde foi amostrado um grande número de espécies com apenas um ou dois indivíduos e um número bem menor de espécies abundantes.

Revista Brasileira de Zoologia 24 (2): 482-492, junho 2007
A riqueza de espécies também é um componente importante da diversidade de espécies de um ecossistema (MagurRan 2004). As riquezas encontradas na Enseada (77) e no Canal do Rio (43) são semelhantes àquelas descritas para outros sistemas aquáticos da bacia do Tocantins. MERONA (1986) encontrou uma riqueza mínima de 48 espécies e máxima de 85 , durante três anos de coleta na região do baixo rio Tocantins. TejerINA-GARro et al. (1998) encontraram 92 espécies em lagos associados ao rio Araguaia e Melo et al. (2003) coletaram 82 espécies em um tributário deste rio.

Comparando-se os dois ambientes estudados, a diversidade de espécies na Enseada foi maior do que a observada no Canal do Rio. Ambientes lóticos parecem apresentar condições variáveis e severas no que diz respeito ao seu regime de fluxo (Allan 1997). Isso exige adaptações e, conseqüentemente, maior gasto energético dos organismos aquáticos para se moverem contra a corrente e para diminuírem a probabilidade de serem arrastados rio abaixo pela correnteza (Moss 1995, Allan 1997). Dessa forma, trechos onde a água é mais lenta ou mesmo parada ao longo do canal do rio, como é caso da Enseada amostrada no Rio das Mortes, representam um ambiente mais estável, onde a ausência de um fluxo forte exige menos gasto de energia dos organismos para sua permanência no local (Allan 1997).

Além disso, a característica lêntica facilita a decantação de fragmentos lenhosos advindos da vegetação marginal. Isso permite a formação de ambientes mais ricos em microhabitats, que são utilizados como abrigo contra predadores por muitas espécies de peixes (Matthews 2003). Os recursos alimentares alóctones, como insetos terrestres, frutos, sementes, folhas e flores, também podem ficar mais tempo disponíveis para os peixes, uma vez que não são rapidamente carreados rio abaixo.

Os resultados deste estudo mostraram que as comunidades nos ambientes amostrados foram variáveis ao longo do ano. Isso é resultado da influência do regime de inundação, que alterna momentos de expansão e retração laterais dos ambientes aquáticos e regula os movimentos dos peixes entre estes ambientes e a planície de inundação (Oliveira \& Garavello 2003).

Durante o período de cheia muitas espécies de peixes tendem a se dispersar ao longo da planície inundada para explorar novos hábitats e recursos alimentares (SAINT-PAUL et al. 2000, Silvano et al. 2000, Galacatos et al. 2004, Arrington et al. 2005). $\mathrm{Na}$ seca, com a diminuição do nível da água, os peixes estão mais concentrados nos ambientes e são mais facilmente capturados pelas redes de espera (Goulding et al. 1988, Silvano et al. 2000). Isso explica os menores valores de diversidade e riqueza de espécies observados durante o período de cheia e os maiores durante a seca na Enseada e no Canal do Rio das Mortes. Estes resultados são semelhantes àqueles encontrados em outros estudos na região amazônica (Goulding et al. 1988, SiLvano et al. 2000, Galacatos et al. 2004).

A densidade relativa da biomassa, dada pela análise de captura por unidade de esforço (CPUE), também sofreu variações entre os períodos sazonais em cada um dos ambientes es- 
tudados. Os valores mínimos e máximos encontrados nestes ambientes estão de acordo com aqueles descritos para outros rios amazônicos, onde foram amostrados valores entre 4,29 a $281,53 \mathrm{~g} / \mathrm{m}^{2} / 24$ horas (SANTOS \& FerReira 1999). Essas variações na densidade relativa de peixes são características de ambientes aquáticos associados a planícies de inundação. Elas também ocorrem em função das flutuações sazonais no nível da água que influenciam os processos migratórios da ictiofauna (Goulding et al. 1988, Silvano et al. 2000). Com o início das chuvas durante a enchente, muitas espécies começam a migrar para regiões de cabeceira ou para a planície inundada em busca de alimento, abrigo e condições adequadas para reprodução (Goulding et al. 1988, Lowe-McConnell 1999). Por isso, neste período a probabilidade de captura de alguns cardumes é maior, aumentando a densidade e o número de indivíduos amostrados nesta época do ano nos ambientes.

Na Enseada e no Canal do Rio das Mortes, durante o mês de dezembro, que corresponde ao período de enchente, foram coletados muitos indivíduos de algumas espécies de maior massa corporal, como Ageneiosus inermis (Linnaeus, 1766), Rhaphiodon vulpinus Agassiz, 1829 e Anodus orinocensis (Steindachner, 1887), além de cardumes de algumas espécies de menor porte, como Psectrogaster amazonica, Auchenipterus nuchalis (Spix \& Agassiz, 1829) e Triportheus auritus. A abundância e a densidade relativa (CPUE) de peixes nestes ambientes durante tal período foram as mais elevadas ao longo do ano de estudo.

Em relação à abundância, a análise de agrupamento demonstrou que a Enseada e o Canal do Rio foram mais similares durante os meses relacionados ao período chuvoso. O mesmo foi observado em relação às características limnológicas analisadas. Como mostram alguns estudos, durante o período de cheia a inundação homogeneíza os ambientes em relação à diversidade de hábitats e as características físicas e químicas da água (Gido et al. 1997, Pouilly et al. 1999, Melo et al. 2003), enquanto que, na seca, com a redução do nível da água, as diferenças entre os ambientes se acentuam. Assim, durante esse período a Enseada e o Canal do Rio foram mais similares em abundância de peixes porque apresentavam condições físicas e químicas semelhantes devido à inundação.

Esses resultados sugerem que o pulso de inundação é a principal força que influencia as características físicas e químicas dos ambientes estudados e, conseqüentemente, ocasiona alterações sazonais na diversidade de espécies, abundância e a densidade relativa de peixes (CPUE). Além disso, o pulso de inundação propicia diferentes graus de similaridade entre os dois ambientes em relação às variáveis limnológicas e à abundância de peixes. Sob a influência da marcada sazonalidade e das características geomorfológicas da região os dois ambientes sustentam uma diversidade de espécies tão elevada quanto os ambientes mais diversos da região amazônica.

As diferenças observadas entre a Enseada e o Canal do Rio em relação à diversidade de espécies, bem como a menor similaridade entre eles em relação à abundância de peixes e às características limnológicas durante o período de seca, sugerem que cada ambiente tem uma participação importante na dinâmica da ictiofauna local. Isso indica que os estudos que visem à elaboração de práticas de manejo e conservação da ictiofauna não devem considerar apenas os ambiente como um todo, mas também as particularidades dos diferentes hábitats para que os resultados sejam mais consistentes.

\section{AGRADECIMENTOS}

Ao Laboratório de Ictiologia e Limnologia, Universidade do Estado de Mato Grosso, Campus de Nova Xavantina pelo apoio financeiro e logístico. À CAPES pela concessão de bolsa de mestrado para E.F. da Silva. À Jane D. Lima pelas sugestões e correções deste manuscrito.

\section{REFERÊNCIAS BIBLIOGRÁFICAS}

Agostinho, A.A; L.M. Bini \& L.C. Gomes. 1997. Ecologia de comunidades de peixes de áreas de influência do reservatório de Segredo. In: A.A. Agostinho \& L.C. Gomes (Eds). Reservatório de Segredo: bases ecológicas para o manejo. Maringá, EDUEM, 387p.

ALLAN, J.D. 1997. Stream ecology: structure and function of running waters. London, Chapman \& Hall, XII +388p.

Arrington, D.A.; K.O. Winemiller. \& C.A. Layman. 2005. Community assembly at the patch scale in a species rich river. Oecologia 144 (1): 157-167.

Bayley, P.B. 1995. Understanding large river-floodplain ecossystems: significant economic advantages and increased biodiversity and stability would result from restoration of impaired systems. Bioscience 45 (3): 153-138.

Brasil. 1981. Projeto Radambrasil. Rio de Janeiro, Ministério das Minas e Energia, Secretaria Geral, 640p.

Diegues, A.C. 2002. Médio Curso do Rio Araguaia e Ilha do Bananal, p. 210-216. In: A.C. Diegues (Ed.). Povos e águas: inventário de áreas úmidas brasileiras. São Paulo, NUPAUB, USP, $2^{\text {a }}$ ed., 597p.

Esteves, F.A. 1998. Fundamentos de limnologia. Rio de Janeiro, Interciência, 602p.

Galacatos, K.; R. Barriga-Salazar \& D.J. Stewart. 2004. Seasonal and habitat influences on fish communities within the Lower Yasuni River basin of the Ecuadorian Amazon. Environmental Biology of Fishes 71: 33-51.

Gido, K.B.; D.L. Propst \& M.C. Molles Jr. 1997. Spatial and temporal variation of fish communities in secondary channel of San Juan River, New Mexico and Utah. Environmental Biology of Fishes 49: 417-434.

Gordon, N.D.; T.A. McMahon \& B.L. Finlayson. 1995. Stream hydrology: an introduction for ecologists. Chichester, John Wiley \& Sons, 562p.

Goulding, M. 1980. The fishes and the forest: explorations in Amazonian natural history. California, University of California Press, XI+280p. 
Goulding, M.; M.L. Carvalho \& E.G., Ferreira. 1988. Rio Negro, rich life in poor water: Amazonian diversity and foodchain ecology as seen thought fish communities. The Hague, SPB Academic Publishing, XI + 200p.

JuNK, W.J. 1980. Áreas inundáveis: um desafio para a limnologia. Acta Amazônica 10 (4): 775-795.

Junk, W.J. 1993. Wetlands of tropical South America, p. 679739. In: D. Whigham; S. Hejny \& D. Dyкyjova (Eds).Wetlands of the world I: inventory, ecology and management. Boston, Dr Junk Publishier.

Junk, W.J.; G.M. SoAres \& F.M. Carvalho. 1983. Distribution of fish species in a lake of the Amazon river floodplain near Manaus (Lago Camaleão), with special reference to extreme oxygen conditions. Amazoniana 7: 397-421.

Junk, W.J.; P.B. Bayley \& R.E. SPARKs. 1989. The flood pulse concept in River Floodplains Systems. Canadian Special Publication Fisheries and Aquatic Science 106: 110-127.

KREBS, C.J. 1989. Ecological methodology. New York, Harper Collins Publishers, XII+654p.

Lowe-McConnel, R.H. 1999. Estudos ecológicos de comunidades de peixes. São Paulo, Universidade de São Paulo, $584 \mathrm{p}$.

MagurRan, A.E. 2004. Measuring biological diversity. Oxford, Blackwell Science, 256p.

Marimon, B.S. \& E.S. Lima. 2001. Caracterização fitofisionômica e levantamento florístico preliminar do Pantanal do Rio das Mortes - Araguaia, Cocalinho, Mato Grosso, Brasil. Acta Botânica Brasilica 15 (2): 213-229.

Matthews, W.J. 2003. Patterns in freshwater fish ecology. Massachusetts, Kluer Academic Publishers, XXII+756p.

Melo, C.E. \& C.P. RöPKe. 2004. Alimentação e Distribuição de Piaus (Pisces, Anostomidae) na Planície do Bananal, Mato Grosso, Brasil. Revista Brasileira de Zoologia 21 (1): 51-56.

Melo, C.E.; F.A. Machado \& V. Pinto-Silva. 2003. Diversidade de Peixes em um Córrego de Cerrado no Brasil Central. Brazilian Journal of Ecology 1/2: 17-23.

Melo, C.E.; J.D. Lima; T.L. Melo \& V. Pinto-Silva. 2005. Peixes do Rio das Mortes: identificação e ecologia das espécies mais comuns. Cuiabá, Central de Textos, 145p.

Merona, B. De. 1986/87. Aspectos Ecológicos da Ictiofauna do Baixo Tocantins. Acta Amazônica 16/17:109-124.

Moss, B. 1995. Ecology of fresh waters: man and medium.
London, Blackwell Science, $2^{\text {nd }}$ ed., $417 p$.

Oliveira, A.K. \& J.C. Garavello. 2003. Fish assemblage composition in a tributary of the Mogi-Guaçu River Basin, southeastern Brazil. Iheringia, Séria Zoologia 93 (2): 127-138.

PinTo-Silva, V. 2002. Manual de análise limnológica: métodos e técnicas. Cuiabá, UFMT, 95p.

Pouilly, M.; C. Ibañez; M. Guttierez \& T. Yunoki. 1999. Funcionamiento ecológico de las lagunas de la zona de inundación del río Mamoré (Beni - Bolivia). Revista Boliviana de Ecología 6: 41-54.

Reis, R.E.; O. Kullander \& C.J. Ferraris-Jr. 2003. Check list of freshwater fishes South and Central América. Porto Alegre, EDIPUCRS, 742p.

Santos, G. \& E.J.G., Ferreira. 1999. Peixes da Bacia Amazônica, p. 345-373. In: R.G. Lowe-McConnel (Ed.). Estudos ecológicos de comunidades de peixes tropicais. São Paulo, Universidade de São Paulo, 584p.

Saint-Paul, U.; J. Zuanon; M.A.V. Correa; M. García; N.N. Fabré; U. Berget \& W.J. Junk. 2000. Fish communities in central Amazonian white and blackwater floodplains. Environmental Biology of Fishes 57: 235-250.

Silvano, R.A.M.; B.D. Amaral \& O.T. Oyakawa. 2000. Spatial and temporal patterns of diversity and distribution of Upper Juruá River fish community (Brazilian Amazon). Environmental Biology of Fishes 57: 25-35.

Sioli, H. 1991. Amazônia: fundamentos da ecologia da maior região de florestas tropicais. Petrópolis, Vozes, $3^{\mathrm{a}}$ ed., $72 \mathrm{p}$.

Siqueira-Souza, F.K. \& C.E.C. Freitas. 2004. Fish diversity of floodplain lakes on the lower stretch of the Solimões river. Brazilian Journal of Biology 64 (3A): 501-510.

Tejerina-garro, F.L.; R., Fortini \& M.A. Rodriguez. 1998. Fish community structure in relation to environmental variation in floodplain lakes of the Araguaia river, Amazon Basin. Environmental Biology of Fishes 51: 399-410.

Vannote, R.L.; G.W. Minshall; K.W. Cummins; J.R. Sedell \& C.E. CusHing. 1980. The River Continuum Concept. Canadian Journal Fisheries Aquatic Science 37: 130-137.

Wetzel, R.G. 1993. Limnologia. Lisboa, Calouste Gulbenkian, 905p.

ZAR, J.H. 1996. Biostatistical analysis. New Jersey, Prentice Hall, $3^{\mathrm{a}}$ ed., 662p.

Recebido em 05.Il.2007; aceito em 05.VI.2007. 